\title{
Chronic activation of CREB and p90RSK in human epileptic hippocampus
}

\author{
Sun Ah Park ${ }^{1,4}$, Tai Seung Kim ${ }^{2}$ \\ Kyeong Sook Choi ${ }^{3}$, Hyun Jin Park \\ Kyoung $\mathrm{Heo}^{1}$ and Byung In Lee $\mathrm{Le}^{1,5}$ \\ ${ }^{1}$ Department of Neurology \\ ${ }^{2}$ Department of Neuropathology \\ Yonsei University College of Medicine \\ 134 Shinchon-dong, Seodaemun-gu \\ CPO Box 8044, Seoul 120-752, Korea \\ ${ }^{3}$ Institute for Medical Sciences \\ Brain Disease Research Center \\ Ajou University School of Medicine, 5 Wonchon-dong \\ Paldal-Gu, Suwon 442-749, Korea \\ ${ }^{4}$ Department of Neurology \\ Soonchunhyang University Bucheon Hospital \\ 1174 Jung-dong, Wonmi-gu, Bucheon 420-767, Korea \\ ${ }^{5}$ Corresponding Author: Tel, 82-2-361-5464; \\ Fax, 82-2-393-0705; E-mail, bilee@yumc.yonsei.ac.kr
}

Accepted 21 August 2003

Abbreviations: AHS, Ammon's horn sclerosis; CA, cornus ammonus; CREB, cyclic AMP regulatory element binding protein; DG, dentate nucleus; ERK, extracellular signal-regulated kinase; GFAP, glial fibrillary acidic protein; MTLE, mesial temporal lobe epilepsy; p90RSK, 90-kDa ribosomal S6 kinase

\begin{abstract}
Mesial temporal lobe epilepsy (MTLE) is associated with severe neuronal death and reactive gliosis in hippocampus. However, the molecular mechanisms underlying these pathological changes remain unanswered. ERK has been reported chronically activated in reactive glia of human epileptic hippocampus. In the present study, we investigated which of the downstream signaling molecules of ERK would be involved in MTLE. Western blot analysis demonstrated that CREB and p90RSK were strongly activated in MTLE patients. Increase in the active forms of CREB and p90RSK resulted not only from the increase in their phosphorylation levels but also from the increase in the protein levels. Activation of CREB and p90RSK was noted in the whole subfields of hippocampus with Ammon's horn sclerosis (AHS) representing a distinctive cellular distribution. However, the common major change was present in proliferating
\end{abstract}

reactive astrocytes. In contrast, their activation was not significant in adjacent temporal lobes despite the presence of a number of astrocytes expressing high levels of GFAP. Our results demonstrate that chronic activation CREB and p90RSK in the epileptic hippocampus may be closely associated with the histopathological changes of AHS.

Keywords: Ammon's horn sclerosis; CREB; ERK; epilepsy; p90RSK

\section{Introduction}

Mesial temporal lobe epilepsy (MTLE) is the most common partial epilepsy, which is frequently resistant to antiepileptic drug therapy. Ammon's horn sclerosis (AHS) is the most common pathological substrate for drug-resistant MTLE, which is characterized by the histopathological changes of hippocampus consisting of neuronal cell death, enhanced neurogenesis, axonal sprouting, and reactive gliosis (Babb and Brown, 1987). However, the molecular mechanisms involved in the genesis and the progression of AHS remain elusive.

Elucidation of the signaling molecules activated abnormally in MTLE patients is indispensable not only for the understanding of its pathogenesis but also for the search of the potential therapeutic target. In human epileptic hippocampal tissue, extracellular signalregulated kinase (ERK) has been reported to be chronically activated in reactive glia (Mandell and VandenBerg, 1999). However, which of the downstream effectors of ERK are involved in this hippocampal pathology has not been defined. Among the possible downstream targets Elk, c-AMP response element binding protein (CREB), and p90RSK were specially evaluated in this study because they have been previously reported to play a critical role in hippocampus as a downstream of ERK in relation to neuronal excitation (Xia, et al., 1996; Angenstein, et al., 1998; Atkins, et al., 1998).

We demonstrate here that cyclic AMP regulatory element binding protein (CREB) and $90-\mathrm{kDa}$ ribosomal S6 kinase (p90RSK) are actively expressed in epileptic hippocampus. Major changes were noted in the proliferating reactive astrocytes rather than in existing neurons. Our results demonstrate that not only the increase in the phosphorylation levels of CREB 
and p90RSK but also the increase in their total expression levels independently of the activated ERK may contribute to their activation.

\section{Materials and Methods}

\section{Hippocampal specimens}

Fourteen surgical specimens from patients with chronic medically refractory MTLE (age: $29 \pm 7$ year-old, duration of epilepsy: $16 \pm 8$ years) were examined. The epileptogenic region was localized to the mesial temporal lobe in all patients by continuous electroencephalography-video monitoring. The concomitantly taken temporal cortex adjacent to hippocampus was also evaluated to investigate the regional differences in six patients. As control specimens, five human control hippocampal specimens were obtained at autopsy, with post mortem intervals ranging from 6-24 $\mathrm{h}$. All tissues were fixed in buffered neutral paraformaldehyde and processed routinely into paraffin, or frozen by liquid nitrogen and subsequently stored in $-70^{\circ} \mathrm{C}$ deep freezer. All procedures were conducted in accordance with consent of the patients or family members, and were approved by the Ethics Committee of the Severance Hospital, Seoul, Korea.

\section{Western blot analysis}

Frozen hippocampal tissue (control $=3$, patients $=6$ ) was homogenized in RIPA buffer. After centrifugation, $60 \mu \mathrm{g}$ of protein was electrophoresed through $12 \%$ acrylamide sodium dodecylsulfate-denaturing gels. The gel was transferred to an immobilon membrane (Millipore, Bedford, MA), and probed with anti-total orphospho specific monoclonal antibody to ERK, CREB, Elk-1, or p90RSK (NEB, Beverly, MA) as recommended by the manufacturer. Detection of the specific protein was performed using the enhanced chemiluminescence system (Amersham Pharmacia Biotech, Buckinghamshire, UK).

\section{Imm unohistochemistry}

All hippocampal specimens were coronally dissected along the septotemporal axis. After deparaffinization in xylene and alchohol, quenching of endogenous peroxidase activity using $1 \%$ hydrogen peroxide and then microwave antigen enhancement (in $10 \mathrm{mM}$ citrate buffer $(\mathrm{pH} \mathrm{6.0)}$ for $10 \mathrm{~min}$ at $1.15 \mathrm{~kW})$ was performed. Subsequently, the slide was incubated with anti-phospho specific monoclonal antibody of ERK $(1: 200)$, CREB $(1: 100)$, or p90RSK $(1: 100)$ in dilution solution (DAKO, Glostrup, Denmark) overnight, or anti-glial fibrillary acidic protein (GFAP) antibody (1: 400; Sigma) for an hour at room temperature. Bio- tinylated anti-rabbit secondary antibody with avidinbiotin protocol (DAKO) was used for detection. Then counterstaining was performed using Hematoxylin.

\section{Quantification of immunoreactive glial cells} of activated CREB and p90RSK in hippocampus For the determination of immunoreactive glial cell densities against respective antibodies all specimens were evaluated. Cell counting was performed in five different areas of hippocampal subfield cornus ammonus (CA) under the light microscope at a magnification of $200 \times$ (Olympus, Tokyo, Japan). Glial nuclei were counted in each unit area of a $500 \mu \mathrm{m} \times$ $500 \mu \mathrm{m}$ rectangle using an ocular grid calibrated with a stage micrometer. Immunoreactive glial cell densities were expressed as a percentage relative to the total number of glial cells in each unit area. The data were analyzed statistically using the SPSS Version 9.0 (SPSS inc., Munich, Germany).

\section{Results}

\section{Assessment of the increased activities of ERK, CREB and p90RSK in human TLE by western blot analysis}

Western blotting using the phospho-specific ERK antibody demonstrated that activation of ERK was noted

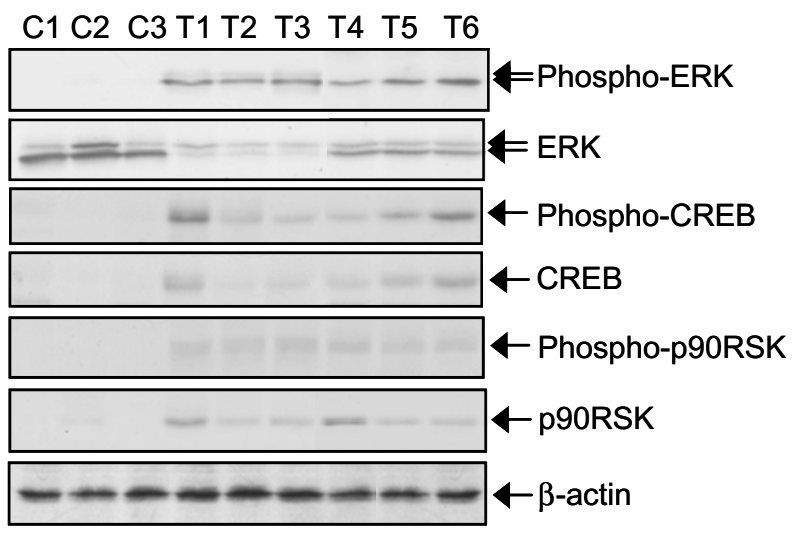

Figure 1. Western blot analysis to detect the activated forms of ERK, CREB, and p90RSK in the hippocampus of the mesial temporal lobe epilepsy (MTLE) patients. Cell extracts were prepared from the hippocampus of three controls and six MTLE patients using homogenizer. A total of $60 \mu \mathrm{g}$ of proteins was loaded for each lane of $12 \%$ SDS-polyacrylamide denaturating gel. Significantly increased activities of phospho-ERK, CREB, and p90RSK activities were detected in epileptic hippocampus but not in normal control. Loading of equal amount of proteins in each lane was confirmed by western blot analysis of $\beta$-actin. $C$, control; $T$, patient with MTLE. 

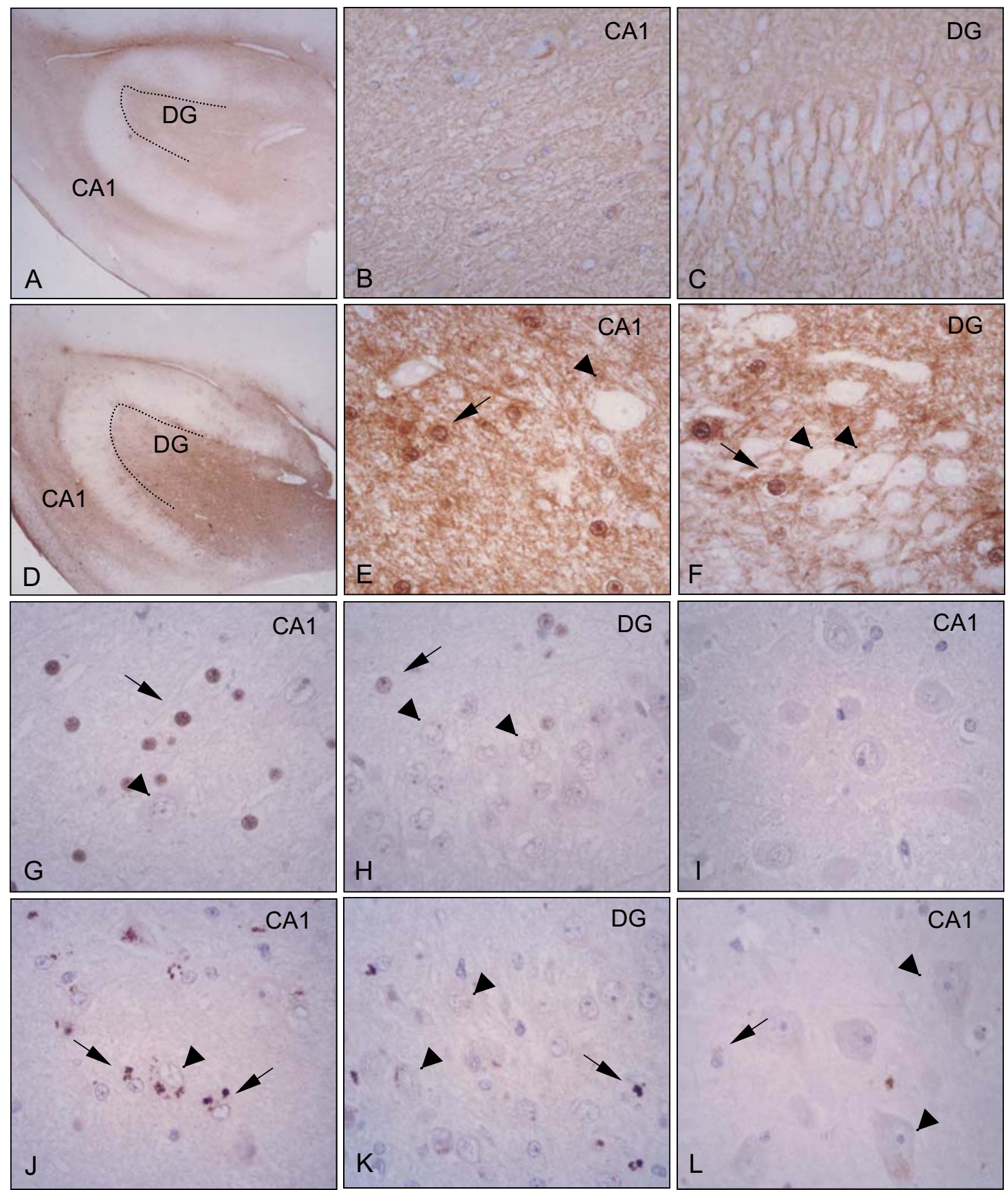

Figure 2. Immunohistochemial detection of GFAP, phospho-ERK, phospho-CREB, and phospho-p90RSK in the epileptic hippocampus and control. (A-C) Low (20x) and higher magnification photomicrograph (400x) of cornus ammonus (CA) and dentate gyrus (DG) region of mesial temporal lobe epilepsy (MTLE) patients shows severe neuronal loss and astrogliosis revealing intense immunoreactivity against GFAP in the fibrillary background and the cytoplasm of reactive astrocytes. (D-F) Its corresponding section demonstrates the profound activation of ERK in all subregions of hippocampus $(20 \mathrm{x})$. Strong immunoreactivities against phospho-ERK were observed both in the process and nucleus of reactive astrocytes (arrows), but not in neurons of CA and DG (arrowheads, 400x). ( $G$ and $H$ ) The corresponding section shows the profound activation of CREB only in the nucleus of reactive astrocytes (arrows), however, no immunoreactivity was detected in the residual neurons in CA and DG (arrowheads, 400). (I) In the histologically normal control hippocampus phospho-CREB staining was not observed (400x). ( $\mathrm{J}$ and $\mathrm{K}$ ) The corresponding section of AHS reveals intense active- p90RSK immunostaining in the perinuclear cytoplasm of both reactive glial cells (arrows) and remaining neurons showing granular appearance in CA and DG subregion (arrowheads, 400x). (L) In control specimen faint and homogenous immunoreactive pattern was observed in neuron (arrowheads) and part of quiescent astrocytes (arrow, 400x). 
only in the specimens of epileptic patients (Figure 1). Interestingly, the expression level of total ERK in MTLE patients' hippocampus was rather lower than that in the control specimen. These results suggest that activation of ERK in MTLE patients might be mainly controlled by phosphorylation. Of the tested possible downstream signaling molecules of ERK pathway, CREB and p90RSK demonstrated a significant increase in their activities when assessed by western blot analysis using the specific antibodies recognizing their respective active form. We were unable to detect any changes in the activities of Elk-1 (data not shown). Interestingly, the total protein levels of CREB and p90RSK were also increased in the epileptic hippocampus. These results suggest that this activation of CREB and p90RSK may be primarily derived from the increase in their protein levels rather than the increase in their activities through phosphorylation. Therefore, our results suggest that both CREB and p90RSK in the AHS may be chronically activated, although their activities may not be exclusively dependent on ERK activities (Figure 1).

\section{Cellular distribution of active ERK, CREB and p90RSK in the epileptic hippocampus}

The characteristic histopathological features of AHS consisting of substantial loss of neurons and increased number of astrocytes were consistently found in all MTLE specimens, which was most extensive in the CA subfield of hippocampus (grade 3 in four and grade 4 in ten patients) (Wyler, et al., 1995). GFAP immunoreactivity was markedly increased in the processes and cytoplasm of astrocytes in the hippocampus from MTLE patients providing the evidence of severe reactive gliosis (Figure $2 \mathrm{~A}-\mathrm{C}$ ). However, in the control hippocampus showing normal morpho- logical features, GFAP expression was only faint (data not shown).

Next, we examined the cellular origins representing high activities of ERK, CREB and p90RSK in MTLE patients' hippocampus by immunohistochemical analysis using the specific antibodies recognizing the actively phosphorylated proteins. While the immuoreactivity of active ERK was observed not only in the nucleus but also in the process of astrocytes (Figure $2 D-F)$, highly active CREB was localized only in the nucleus of the reactive astrocytes in the epileptic hippocampus (Figure $2 \mathrm{G}$ and $\mathrm{H}$ ). None of the existing neurons in $C A$ and dentate gyrus (DG) revealed any immunoreactivity for ERK or CREB. These results suggest that CREB may play a role in the astrogliosis leading to AHS.

In contrast, immunohistochemical analysis to detect the active form of p90RSK demonstrated intense activity of p90RSK in the perinuclear cytoplasm of the reactive glial cells (Figure $2 \mathrm{~J}$ and $\mathrm{K}$ ). Interestingly, active p90RSK immunoreactivity with granular morphology was also noted in the cytoplasm of the remaining neurons. On the other hand, a diffuse pattern of immunoreactivity against active p90RSK but with a much less intensities was detected in the majority of neurons and some quiescent astrocytes of control hippocampal specimen (Figure 2L). These results suggest that p90RSK activity in the existing neurons may be maintained at a considerable level in both normal and epileptic hippocampus. However, the quantitative analysis of the cells expressing activated p90RSK protein revealed a much higher proportion of immunopositive glial cells in the CA region from the MTLE patients than the control (Figure 3). These results seem to suggest a link between the increased p90RSK activity and reactive gliosis of epileptic hippocampus, and its possible involvement in the path-
A

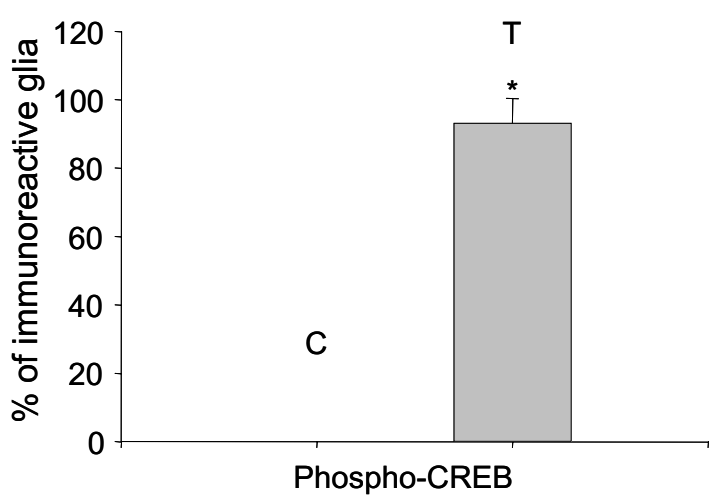

B

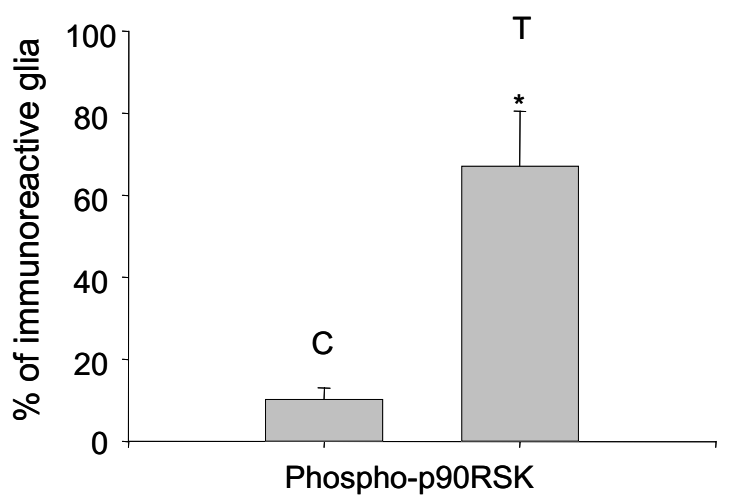

Figure 3. ( $A$ and $B)$ The quantitative analysis of the immunoreactivity against phospho-CREB and p90RSK antibody. The proportion of immunopositive glial cells of activated CREB and p90RSK among total number of counted glia in the observed cornus ammonus (CA) region was markedly increased in mesial temporal lobe epilepsy (MTLE) patients. ${ }^{*} P<0.05$. C, control; T, patient with MTLE. 


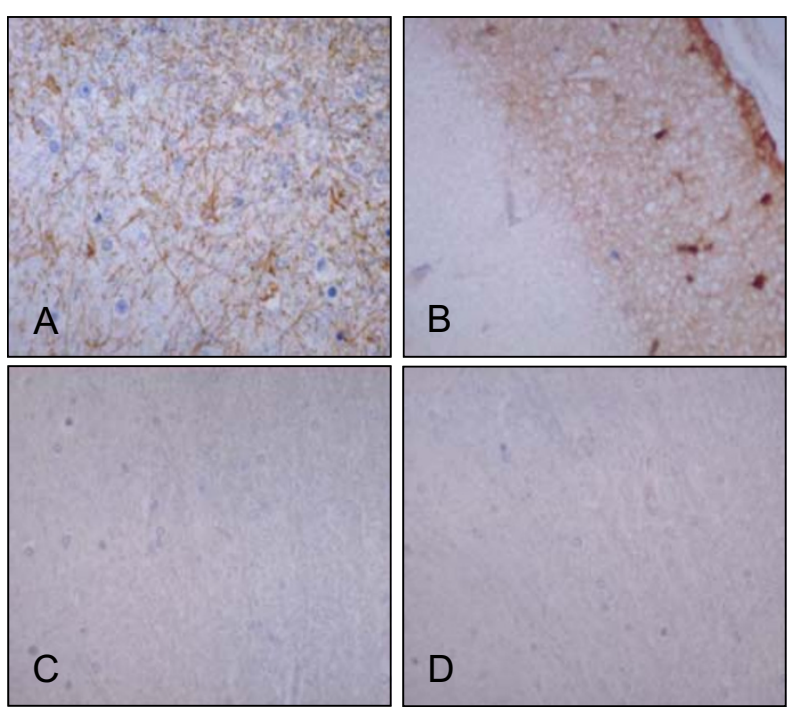

Figure 4. Immunohistochemical detection of GFAP, phospho-ERK, phospho-CREB, and phospho-p90RSK in the adjacent lateral temporal lobe $(200 x)$. (A) In the examined lateral temporal cortex, inferior temporal gyrus, the majorities of the astrocytes both in gray and white matter were strongly reactive with anti-GFAP antibody. (B) However, phospho-ERK immunoreactivity was only noted in the limited area, subpial region. ( $C$ and $D$ ) Phospho-CREB and phosphop90RSK did not demonstrate a detectable level of immunoreactivity.

ogenesis of AHS.

\section{Distribution in the adjacent temporal lobe}

To investigate whether activation of ERK, CREB or p90RSK is regionally specific for the epileptic hippocampus with sclerosis, the immunoreactivitiy of these signaling molecules were examined in six histologically normal adjacent lateral temporal lobes (inferior temporal gyrus) of MTLE patients. The majority of the astrocytes both in gray and white matter were reactive with anti-GFAP antibody (Figure 4A). However, the activated form of ERK was detectable only in subpial astrocytes (Figure 4B). In addition, CREB and p90RSK did not show any detectable extent of activation (Figure $4 \mathrm{C}$ and D). Taken together, our results suggest that chronic activation of ERK, CREB, and p90RSK may be closely associated with the ongoing neuropathologic process for epileptic hippocampus.

\section{Discussion}

The identification of signal transduction events associated with MTLE in hippocampus is very important both for understanding of the pathomechanisms involved in AHS and for designing future therapeutic interventions.

In human epileptic hippocampal tissue ERK has been reported to be chronically activated in reactive glia (Mandell and VandenBerg, 1999). ERK has been known to regulate diverse functions, such as cell growth, proliferation, differentiation, and apoptosis (Schaeffer and Weber, 1999). Moreover, ERK is an integral component of cellular response to glutamatergic stimulation (Bading and Greenberg, 1991; Fiore, et al., 1993), and mediates seizure-induced hippocampal neuronal death in in vitro epilepsy model (Murray, et al., 1998).

Consistent with the previous report by Mandell and WandenBerg (1999), we also observed a significant activation of ERK in the hippocampus of MTLE patients. In the present study, we clearly demonstrated that the primary underlying mechanism leading to ERK activation is the increase of phosphorylation level, since the total expression of ERK in control specimens was higher than that in MTLE patients. Among the tested signaling molecules associated with ERK pathway, both CREB and p90RSK revealed a significant activation in the hippocampus, indicating their possible role in the pathogenesis of AHS. However, the activation mechanism of CREB and p90RSK may not be exclusively dependent on the activated ERK. Compared with the control specimens, the expression levels of total CREB and p90RSK protein were significantly elevated in the hippocampus of MTLE patients. Especially the expression pattern of total CREB protein was almost paralleled with the activation profile of CREB in western blot analysis using hippocampal homogenates. While ERK, CREB and p90RSK were all activated in astrocytes, significantly high activity of p90RSK was also localized in neurons. These results suggest that another activation mechanism of CREB and p90RSK may exist independently of ERK pathway, possibly through the transcriptional or translational control. It was unexpectable that activation of CREB and p90RSK were not identical to that of ERK, because CREB has been known to be activated ERK-dependently in neuronal excitatory gene expression (Sheng et al., 1990; Xia et al., 1996) and p90RSK be usually located downstream of the ERK pathway in response to virtually all extrcellular signaling molecules that stimulate the ERK pathway (Blenis, 1993).

The significance of reactive astrocytes in the epileptogenesis has been raised recently. They regulate neuronal excitability not only by the clearance of extracellular potassium and glutamate but also by the release of inhibitory transmitters. Furthermore, they promote the mobilization of intracellular calcium leading to more neuronal damage and formation of dystrophic neurites by the expression of molecules with high neurite promoting activity (Duffy and MacVicar, 
1999; Grisar, et al., 1999; Heinemann, et al., 1999). However, the signaling molecules involved in the reactive gliosis associated with epilepsy have not been extensively investigated. Our immunohistohemical study demonstrated that the activation of CREB and p90RSK in hippocampus of MTLE patients might be closely associated with reactive gliosis. Both CREB and p90RSK demonstrated a notable activation in glial cells of MTLE patients. In chronic epilepsy, excessive neuronal activation by epileptic discharges renders cells exposed to the immoderate amount of glutamate and calcium resulting in the execution of neuronal cell death and synaptic sprouting (Heinemann, et al., 1986; Olney, et al., 1986). CREB has been reported to be a critical mediator of calciumand glutamate-dependent gene expression (Sheng, et al., 1990; Xia, et al., 1996). However its function in astrocytes has not been investigated. In this study, the active form of CREB was exclusively localized in the nuclei of astrocytes of epileptic hippocampus, suggesting its possible role as a transcription factor (Martin, et al., 1997). Interestingly, intense immunoreactivity of phospho-p90RSK with granular morphology was noted in the glial cytoplasm of MTLE patients in our study. Previously, Angenstein et al (1998) has reported that glutamate initiated translocation of p90RSK to polyribosomes for protein synthesis in hippocampus. Therefore, it is expected that p90RSK may play a possible role in human epileptic hippocampus via regulation of protein synthesis. However, to further elucidate the significance of chronic activation of CREB and p90RSK in epileptic hippocampus and their relationship with ERK pathway, and to evaluate the ERK pathway as the potential therapeutic target of MTLE, further studies using animal models and in vitro experiments would be required.

\section{Acknowledgement}

This work was supported by a grant from the Ministry of Health and Welfare (02-PJ1-PG3-21301-0019, to BI Lee) and from the KOSEF/BDRC Ajou University (R11-1998-052-08005, to KS Choi).

\section{References}

Angenstein F, Greenough WT, Weiler IJ. Metabotropic glutamate receptor-initiated translocation of protein kinase p90rsk to polyribosomes: a possible factor regulating synaptic protein synthesis. Proc Natl Acad Sci USA 1998;95: 15078-80

Atkins CM, Selcher JC, Petraitis JJ, Trzaskos JM, Sweatt JD. The MAP kinase cascade is required for mammalian associative learning. Nature Neurosci 1998;1:602-9
Babb TL, Brown WJ. Pathological findings in epilepsy. In Surgical treatment of the epilepsies (Engel J, Jr, ed), 1987, 511-540, Raven press, New York NY

Bading $H$, Greenberg ME. Stimulation of protein tyrosine phosphorylation by NMDA receptor activation. Science 1991; 253:912-4

Blenis J. Signal transduction via the MAP kinases: proceed at your own RSK. Proc Natl Acad Sci USA 1993;90:5889-92

Duffy S, MacVicar BA. Modulation of neuronal excitability by astrocytes. Adv Neurol 1999;79:573-81

Fiore RS, Murphy TH, Sanghera JS, Pelech SL, Baraban JM. Activation of p42 mitogen-activated protein kinase by glutamate receptor stimulation in rat primary cortical cultures. J Neurochem 1993;61:1626-33

Grisar T, Lakaye B, Thomas E, Buttendorf L, Minet A. The molecular neuron-glia couple and epileptogenesis. Adv Neurol 1999;79:591-601

Heinemann U, Konnerth A, Pumain R, Wadman WJ. Extracellular calcium and potassium concentration changes in chronic epileptic brain tissue. Adv Neurol 1986;44:641-62

Heinemann U, Gabriel S, Schuchmann S, Eder C. Contribution of astrocytes to seizure activity. Adv Neurol 1999;79: 583-90

Mandell JW, VandenBerg SR. ERK/MAP kinase is chronically activated in human reactive astrocytes. Neuroreport 1999;10: 3567-72

Martin KC, Michael D, Rose JC, Barad M, Casadio A, Zhu $\mathrm{H}$, Kandel ER. MAP kinase translocate into the nucleus of the presynaptic cell and is required for long-term facilitation in Aplysia. Neuron 1997;18:899-912

Murray B, Alessandrini A, Cole AJ, Yee AG, Furshpan EJ. Inhibition of the p44/42 MAP kinase pathway protects hippocampal neurons in a cell-culture model of seizure activity. Proc Natl Acad Sci USA 1998;95:11975-80

Olney JW, Collins RC, Sloviter RS. Excitotoxic mechanisms of epileptic brain damage. Adv Neurol 1986;44:857-78

Schaeffer HJ, Weber MJ. Mitogen-activated protein kinases: specific messages from ubiquitous messengers. Mol Cell Biol 1999;19:2435-44

Sheng M, McFadden G, Greenberg ME. Membrane depolarization and calcium induce $c$-fos transcription via phosphorylation of transcription factor CREB. Neuron 1990;4:57182

Wyler AR, Hermann BP, Somes G. Extent of medial temporal resection on outcome from anterior temporal lobectomy: a randomized prospective study. Neurosurgery 1995; 37:982-91

Xia Z, Dudek H, Miranti CK, Greenberg ME. Calcium influx via the NMDA receptor induces immediate early gene transcription by a MAP kinase/ERK-dependent mechanism. J Neurosci 1996;16:5425-36 